\title{
La Val Maira (Piemonte): laboratorio territoriale di un nuovo popolamento montano
}

\section{Giacomo Pettenati}

\section{(2) OpenEdition}

1 Journals

\section{Édition électronique}

URL : https://journals.openedition.org/rga/2201

DOI : $10.4000 /$ rga.2201

ISSN : $1760-7426$

Traduction(s) :

Maira Valley (Piedmont): a territorial laboratory of a new mountain population - URL : https://

journals.openedition.org/rga/2208 [en]

Éditeur :

Association pour la diffusion de la recherche alpine, UGA Éditions/Université Grenoble Alpes

Référence électronique

Giacomo Pettenati, « La Val Maira (Piemonte): laboratorio territoriale di un nuovo popolamento montano », Journal of Alpine Research | Revue de géographie alpine [En ligne], 101-3 | 2013, mis en ligne le 25 mars 2014, consulté le 08 décembre 2022. URL : http://journals.openedition.org/rga/2201 ; DOI : https://doi.org/10.4000/rga.2201

Ce document a été généré automatiquement le 8 décembre 2022.

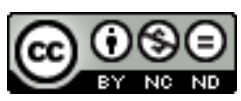

Creative Commons - Attribution - Pas d'Utilisation Commerciale - Pas de Modification 4.0 International - CC BY-NC-ND 4.0

https://creativecommons.org/licenses/by-nc-nd/4.0/ 


\title{
La Val Maira (Piemonte): laboratorio territoriale di un nuovo popolamento montano
}

\author{
Giacomo Pettenati
}

\begin{abstract}
"Primo: le Alpi costituiscono un ambiente e una cultura da salvare non solo per l'Europa, ma per tutta l'umanità; secondo: le Alpi non si possono salvare se non si salvano anche i popoli "montanari" che vivono "nella"montagna e "della"montagna; terzo: "montanari" non si nasce, ma si diventa: oggi nel farsi "montanari" nella Alpi è cruciale l'assunzione di responsabilità della "custodia" dell'integrità dell'ambiente naturale alpino in nome e per conto dell'umanità intera" (Zanzi, 2004, p. 341).
\end{abstract}

Queste riflessioni dello storico lombardo Luigi Zanzi sintetizzano efficacemente l'importanza di prendere in considerazione oggi in modo nuovo le relazioni tra i territori montani e le popolazioni che li abitano, superando una visione superficiale dell'economia e della società alpine, viste spesso come irrimediabilmente marginali ed arretrate rispetto alle aree urbane, alle pianure o ai poli turistici. In molte vallate italiane, come già avvenuto in Francia alcuni decenni fa, è infatti sempre più rilevante il ruolo dei "nuovi montanari" ai quali si riferisce Zanzi, la cui presenza, grazie al loro approccio attivo nei confronti del territorio e delle sue risorse, smorza gli effetti di dinamiche demografiche ancora negative. Ciò avviene grazie a un nuovo (dal punto di vista qualitativo) popolamento della montagna, che viene riterritorializzata da nuove reti e nuove progettualità, le quali portano innanzitutto ad una restituzione di dignità a territori, che lentamente cercano di superare la propria subalternità nei confronti della città.

Questo contributo si propone di approfondire il ruolo dei nuovi abitanti nelle dinamiche territoriali della Val Maira, una delle valli simbolo del nuovo popolamento montano sul versante italiano delle Alpi occidentali (Bartaletti, 2004; Dematteis, 2011), attraverso i risultati di una serie di ricerche nell'ambito delle quali sono stati intervistati 36 "nuovi abitanti" della valle. Come tali si intendono persone adulte che si sono volontariamente trasferite in maniera stabile in un comune della valle. Le 
interviste, pur realizzate nell'ambito di diverse ricerche $^{1}$, riguardavano prevalentemente le motivazioni del trasferimento e le relazioni tra i soggetti coinvolti ed il proprio nuovo territorio di vita e sono state effettuate tra il 2009 ed il 2013, nei comuni di Canosio, Stroppo, Acceglio e San Damiano Macra.

\section{Spopolamento e nuovo popolamento nelle Alpi occidentali italiane}

3 A partire dalla fine del XIX secolo le Alpi occidentali hanno conosciuto una rivoluzione demografica senza precedenti, che ha sconvolto il delicato equilibrio tra popolamento, risorse ed ambiente che caratterizzava abbastanza stabilmente questa porzione del territorio alpino fin dal Medioevo (Bätzing, 2005). In pochi decenni la maggior parte dei comuni montani delle regioni alpine italiane e francesi ${ }^{2}$ ha infatti perso grandi percentuali della propria popolazione, soprattutto tra le fasce più giovani e attive, che hanno abbandonato le proprie terre d'origine in favore dei comuni di fondovalle, delle città industrializzate di pianura o di paesi stranieri. L'emigrazione stagionale di alcuni gruppi di popolazione rappresentava da sempre un espediente utilizzato dalle comunità alpine per fare fronte all'eccessiva densità demografica, soprattutto nei mesi invernali, quando la rigidità del clima e la conseguente riduzione della disponibilità di risorse e di opportunità lavorative determinava una situazione di disoccupazione forzata, definita da alcuni chômage hivernal (Viazzo, 1989). In questo periodo l'emigrazione stagionale diventa però definitiva, trasformandosi da strumento di compensazione temporanea del rapporto popolazione-risorse a componente chiave di un imminente declino sociale ed economico (Salsa, 2007), segnando secondo molti la fine della civiltà tradizionale alpina (Camanni, 2002), la progressiva marginalizzazione dei territori montani e la trasformazione di molte vallate in quello che lo scrittore cuneese Nuto Revelli (1977) ha definito il mondo dei vinti. Tra le cause principali di questo fenomeno si possono senza dubbio individuare la massiccia industrializzazione dei fondovalle e lo sviluppo dell'agricoltura industriale di pianura che hanno reso fortemente marginali le economie montane (Bätzing, 2005); a ciò è necessario aggiungere, soprattutto in riferimento all'emigrazione avvenuta a partire dai primi decenni del ' 900 , le forti sollecitazioni culturali provenienti dal modello di vita moderno di matrice urbana (Salsa, 2007).

4 A partire dagli anni '70 del secolo scorso, pur mantenendosi la maggior parte delle ragioni strutturali dello spopolamento della montagna più marginale, hanno iniziato a manifestarsi interessanti episodi di inversione di tendenza, soprattutto in alcune regioni montane rurali delle Alpi francesi, come il Beaufortain (Fourny 1994) o il Diois (Cognard, 2006) $)^{3}$. In Italia questo nuovo popolamento delle aree montane rurali ha fatto la propria apparizione intorno alla metà degli anni ' 80 , prevalentemente, per quanto riguarda le Alpi occidentali, nelle vallate cuneesi (Val Maira, Val Varaita, Valle Po), quelle maggiormente colpite dalla massiccia emigrazione dei decenni precedenti (Bartaletti, 2004; Dematteis, 2011).

5 Dal momento che le aree montane interessate dal fenomeno del ripopolamento mantengono il proprio stato di marginalità sociale e soprattutto economica, rispetto alle vallate turistiche ed alle vicine pianure (Crescimanno et al., 2010), rispetto alle quali continuano ad offrire meno opportunità lavorative, i protagonisti di queste dinamiche possono essere ricondotti alla categoria dei cosiddetti amenity migrants (Moss, 2006; 
Perlik, 2006), ovvero persone che decidono di trasferirsi in un contesto non urbano (in questo caso montano) grazie al suo elevato valore ambientale, al quale si ritiene venga associata un'altrettanto alta qualità di vita ${ }^{4}$. Ad essi vengono solitamente contrapposte altre tipologie di nuovi residenti dei territori montani, come gli gli economic migrants (Chipeniuk, 2004) o necessitati (Dematteis, 2011) che decidono di insediarsi in un territorio montano per ragioni prevalentemente economiche; oppure $\mathrm{i}$ third-age migrants (Perlik, 2006), che si trasferiscono in montagna una volta raggiunta l'età della pensione.

6 Dal punto di vista delle dinamiche territoriali, questi nuovi abitanti manifestano spesso un approccio attivo al territorio, ovvero una territorialità attiva, frutto della scelta consapevole di vivere in montagna, che porta ad atteggiamenti e progettualità molto diversi da quelli di chi, nato e cresciuto in queste aree, ha a volte la tendenza a subirne inerzialmente i disagi (Salsa, 2007). La loro scelta di trasferirsi in montagna è infatti spesso accompagnata da un progetto, di vita o lavorativo, del quale il territorio di nuova residenza rappresenta una componente fondamentale (Cognard, 2006).

Per queste ragioni molti osservatori attribuiscono ai protagonisti di questo nuovo popolamento alpino un ruolo determinante nell'invertire "la spirale della marginalità" (Buran et al., 1998) che colpisce molte valli. In quest'ottica il futuro della montagna sarebbe in buona parte affidato a questi nuovi montanari, che si assumono la responsabilità della tutela dell'ambiente naturale e culturale alpino, opponendo una resistenza esistenziale alle difficoltà della vita in montagna (Zanzi, 2004) e mettendo in gioco "in modo innovativo risorse territoriali precedentemente utilizzate in modo tradizionale nel mondo alpino" (Corrado 2011, p. 19). Come osserva Magnaghi (2000, p. 90), infatti, "lo sviluppo locale ha il suo rito di fondazione nel riprendersi cura dei luoghi a partire da nuove culture, da nuovi soggetti, da nuovi abitanti e nuovi produttori, che li reinterpretano, si appropriano di saperi e paesaggi, trasformandoli attraverso la contaminazione con culture diverse".

\section{Il contesto territoriale: la Val Maira}

8 La Valle Maira si sviluppa con andamento est-ovest per circa $60 \mathrm{~km}$ nelle Alpi Cozie cuneesi, dal fondovalle pianeggiante della cittadina di Dronero, punto di riferimento funzionale della valle, fino allo spartiacque italo-francese dei monti Chambeyron e Sautron, che la separa dalle valli francesi dell'Ubaye e dell'Ubayette, con le quali è stata storicamente unita attraverso il Col Maurin. La maggior parte dell'asse vallivo è infatti caratterizzato da una morfologia particolarmente angusta, al punto che solo nel XVII secolo è stato possibile ricavare lo spazio per una strada carrozzabile che collegasse la bassa valle e la pianura piemontese con l'alta valle fino ad allora strettamente legata alle vallate transalpine.

9 Dal punto di vista amministrativo, il territorio della Val Maira è suddiviso in quattordici comuni, tutti tranne uno (Busca) classificati come montani, secondo le definizioni legislative italiane ${ }^{5}$. Anche la distribuzione dell'insediamento segue la movimentata morfologia della valle, con ben sette capoluoghi comunali collocati oltre i 1000 metri di altitudine (Prazzo, Stroppo, Acceglio, Canosio, Marmora ed Elva).

10 Come mostra la tabella 1, la distribuzione della popolazione nella valle presenta oggi un grande squilibrio in favore dei quattro grandi comuni del fondovalle, dove oggi si concentra oltre 1 ' $80 \%$ della popolazione complessiva della valle, grazie anche 
all'emigrazione più recente, della quale Roccabruna, Busca, Villar San Costanzo e Dronero sono state destinazioni privilegiate. Gli altri comuni della valle hanno tutti meno di 200 residenti, con la sola eccezione di San Damiano Macra, che si attesta poco oltre i 400 abitanti (dati Istat, 2011).

Tabella 1. Evoluzione demografica dei comuni della Val Maira

\begin{tabular}{|l|l|l|l|l|l|l|l|l|l|}
\hline Comune & Altitudine slm (capoluogo) & 1931 & 1951 & 1961 & 1971 & 1981 & 1991 & 2001 & 2011 \\
\hline Acceglio & $1200 \mathrm{~m}$ & 1254 & 911 & 658 & 509 & 355 & 238 & 197 & 174 \\
\hline Prazzo & $1030 \mathrm{~m}$ & 1275 & 1029 & 787 & 516 & 360 & 282 & 218 & 175 \\
\hline Canosio & $1323 \mathrm{~m}$ & 399 & 270 & 241 & 162 & 136 & 106 & 93 & 82 \\
\hline Marmora & $1223 \mathrm{~m}$ & 645 & 446 & 353 & 211 & 173 & 140 & 99 & 74 \\
\hline Stroppo & $1087 \mathrm{~m}$ & 982 & 635 & 462 & 262 & 186 & 124 & 108 & 107 \\
\hline Macra & $875 \mathrm{~m}$ & 861 & 582 & 349 & 155 & 107 & 81 & 61 & 52 \\
\hline Celle di Macra & $1270 \mathrm{~m}$ & 1194 & 758 & 528 & 216 & 193 & 147 & 105 & 93 \\
\hline San Damiano Macra & $743 \mathrm{~m}$ & 3208 & 2149 & 1524 & 1017 & 690 & 548 & 477 & 439 \\
\hline Cartignano & $704 \mathrm{~m}$ & 817 & 440 & 357 & 241 & 204 & 177 & 170 & 178 \\
\hline Elva & $1637 \mathrm{~m}$ & 801 & 556 & 396 & 252 & 199 & 154 & 114 & 94 \\
\hline Dronero & $622 \mathrm{~m}$ & 7950 & 6615 & 6670 & 7107 & 7124 & 6969 & 7012 & 7205 \\
\hline Roccabruna & $640 \mathrm{~m}$ & 2370 & 1865 & 1506 & 1208 & 1177 & 1308 & 1460 & 1589 \\
\hline Busca & $500 \mathrm{~m}$ & 8895 & 8458 & 7787 & 7851 & 8182 & 8913 & 9469 & 10049 \\
\hline Villar San Costanzo & 605 & 1910 & 1641 & 1451 & 1223 & 1207 & 1396 & 1502 \\
\hline
\end{tabular}

Fonte: Istat

11 La Val Maira ha infatti vissuto una contrazione demografica avviatasi all'inizio del XX secolo nei comuni dell'alta valle e che si è protratta fino a pochi anni fa, senza soluzione di continuità. Il decremento di popolazione ha portato tutti i comuni della media ed alta valle a perdere percentuali impressionanti di popolazione che oscillano tra l' $80 \% \mathrm{di}$ Canosio e il $94 \%$ di Macra. Diversa anche in questo caso è la situazione dei comuni di fondovalle, che hanno invece mantenuto sostanzialmente stabile la propria popolazione (figura 1). 
Figura 1. L'evoluzione demografica dei comuni della Val Maira, rispetto alla popolazione del 1931 $(=100 \%)$

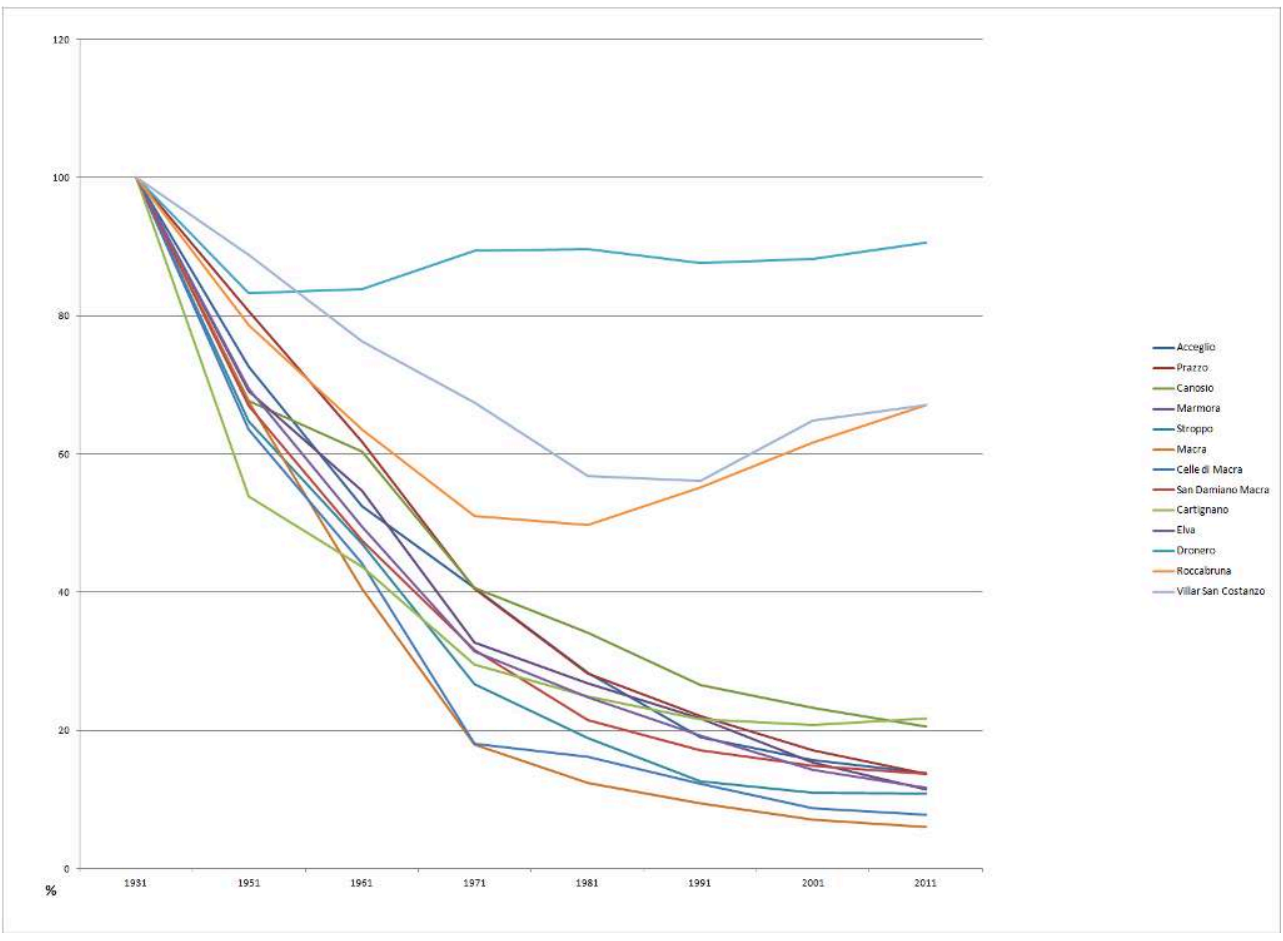

Fonte: dati Istat

1 malessere demografico (Golini et al., 2000) vissuto dalla valle ha determinato importanti ripercussioni economiche, al punto che oggi viene definita come un'area molto marginale dai principali studi socio-economici sulla montagna italiana (Crescimanno et al., 2010). La residua economia locale si fonda prevalentemente su agricoltura ed allevamento (bovini, ovini e caprini), turismo estivo ed invernale non "di massa" (escursionismo, scialpinismo) e settore edile (Regione Piemonte, 2012). La scarsa pressione antropica sopraggiunta negli ultimi decenni e la scarsa accessibilità hanno inoltre portato ad un progressivo aumento della wilderness e della qualità ambientale della valle che oggi viene definita come "area eco sistemica di elevata integrità $e$ bellezza" (Crescimanno et al., 2010).

Dal punto di vista culturale, la Val Maira, insieme alla maggior parte delle altre vallate alpine piemontesi, a partire dagli anni '70 del XX secolo è stata scenario di una riscoperta (o scoperta ex novo, come sostengono alcuni) dell' identità linguistica e culturale occitana, che da allora è diventata la componente fondamentale di un'identità territoriale utilizzata anche in chiave politica e di sviluppo territoriale (Pla-Lang, 2008)

\section{Le ricerche: nuovi abitanti in Val Maira}

Come già osservato, le stesse caratteristiche che hanno determinato la condizione di marginalità della valle (scarsa pressione turistica, elevata qualità ambientale, società rurale) si sono rivelate nel tempo importanti fattori attrattivi per un lento ma costante ritorno di abitanti, che nel giro di pochi decenni ha modificato le traiettorie di sviluppo del territorio, rendendolo una delle aree alpine italiane nelle quali si manifesta con la maggiore evidenza - e con notevoli elementi di interesse - la tendenza al nuovo 
popolamento alpino discussa nel primo paragrafo (Camanni, 2002; Bartaletti, 2004; Dematteis, 2011).

Le prime manifestazioni di questo fenomeno si sono avute all'inizio degli anni ' 80 , quando in Val Maira, come nelle vicine valli Grana, Varaita, Po e Stura, arrivarono gruppi di giovani provenienti in prevalenza dalle città della pianura piemontese (Torino e Cuneo), mossi da legami familiari con le montagne cuneesi, dalla riscoperta dell'identità occitana, che in quegli anni si stava affermando in Piemonte, e dalla volontà politica di ricercare modelli di vita alternativi a quelli della città industriale. A questi pionieri hanno fatto seguito in maniera costante nel corso del tempo decine di nuovi nuclei familiari, provenienti in maggior parte dal Piemonte, ma anche da altre parti d'Italia (Lombardia, Liguria) ed Europa (Germania, Austria, Svizzera), al punto che oggi in alcuni comuni della valle tra gli adulti in età lavorativa i non autoctoni costituiscono la maggioranza. Questa incidenza dei nuovi abitanti sul totale della popolazione può portare a considerare la Val Maira una sorta di "laboratorio territoriale" 6 , nel quale osservare le caratteristiche e gli effetti del nuovo popolamento delle aree marginali alpine.

È necessario sottolineare come questo ripopolamento della valle non sia tanto da considerarsi in termini quantitativi, a causa dell'inerzia demografica dovuta all'elevata età media della popolazione, che rende poco evidente l'inversione di tendenza, quanto in termini qualitativi, dal momento che quasi tutti gli intervistati possono essere considerati "portatori di progetti" (Cognard, 2006), personali e lavorativi, dei quali il territorio montano e le sue risorse sono una componente chiave.

17 La tabella 2 sintetizza le caratteristiche delle 36 persone intervistate, tra il 2008 ed il 2013, nel corso delle ricerche che hanno portato alle riflessioni contenute in questo contributo. Il paragrafo successivo approfondisce invece alcuni aspetti della relazione tra i nuovi abitanti ed il territorio della Val Maira, sottolineando il loro ruolo nell'indirizzare le traiettorie sociali, economiche e culturali della valle.

Tabella 2. Sintesi dei risultati delle interviste a 36 "nuovi abitanti" della Val Maira

\begin{tabular}{|c|c|c|}
\hline \multicolumn{3}{|c|}{ Totale intervistati: 36} \\
\hline Età & $20-30$ & 4 \\
\hline & $30-40$ & \\
\hline & $40-50$ & 18 \\
\hline & $50-60$ & 8 \\
\hline & $60-70$ & 2 \\
\hline \multirow{3}{*}{ Provenienza } & Piemonte & 24 \\
\hline & Resto d'Italia & 6 \\
\hline & Estero & 6 \\
\hline Settore professionale & Servizi (scuola, sanità, etc.) & 7 \\
\hline
\end{tabular}




\begin{tabular}{|c|c|c|}
\hline & Artigianato & 5 \\
\hline & Agricoltura/Allevamento & 8 \\
\hline & Edilizia & 5 \\
\hline & Turismo (ricettività, ristorazione, etc.) & 16 \\
\hline & Attività associativa legata al territorio & 6 \\
\hline & Attività politica & 4 \\
\hline innovazione & $\begin{array}{l}\text { Agricoltura innovativa (biologico, multifunzionale, } \\
\text { ippoturismo, etc.) }\end{array}$ & 6 \\
\hline & Telelavoro & 2 \\
\hline
\end{tabular}

\section{Gli effetti territoriali del nuovo popolamento della Val Maira}

\section{La territorialità attiva dei nuovi abitanti}

La territorialità ${ }^{7}$ di molti nuovi abitanti della valle si può definire come una territorialità attiva, nella quali i soggetti sono "in grado di agire, di rivestire ruoli e di svolgere azioni innovative, configurando, in questo modo, strategie di risposta/ resistenza rispetto a quelle impositive del controllo e costruendo, così, cambiamenti e innovazioni" (Governa, 2005, p. 59). Questo approccio attivo all'agire quotidiano è dimostrato dal fatto che i nuovi abitanti sono protagonisti in due ambiti fondamentali della vita del territorio, quello politico e quello economico. Prendendo ad esempio il comune di Stroppo, entrambi i candidati sindaco alle elezioni comunali del 2009 erano nuovi abitanti, come anche la maggioranza (9/12) dei consiglieri comunali eletti in quell'occasione. A questa attività politica formale si affianca una fervente attività nell'ambito politica cosiddetta informale (Painter and Jeffrey, 2009), testimonianza ad esempio dalla frequente partecipazione alla creazione di associazioni locali dedicate alla promozione del territorio (es. pro loco) o ad attività di affermazione del nuovo presidio territoriale del quale sono protagonisti (es. progetti di sviluppo del territorio, dibattiti sull'abitare in montagna, etc.). Molti di essi sono protagonisti di quella che si potrebbe definire una progettualità territoriale, che integra le prospettive professionali e famigliari dei singoli individui con progetti, spesso di scala sovra locale, che mirano a valorizzare il territorio d'insediamento scelto da questi soggetti ${ }^{8}$.

Un'ulteriore dimostrazione dell'approccio attivo dei nuovi abitanti nei confronti del territorio è offerta dalle caratteristiche delle loro scelte lavorative. Le attività gestite dai nuovi abitanti sono spesso caratterizzate da buoni livelli di innovazione, in relazione al contesto socio-economico in cui si situano, tanto nel periodo di arrivo dei primi nuovi insediati, impegnati in attività turistiche fondate ad esempio sull'ippoturismo e sulla multifunzionalità agricola, molto rare a quell'epoca, quanto oggi, come dimostrano i numerosi esempi di agricoltura biologica, di utilizzo delle 
nuove tecnologie (telelavoro) e di strutture turistiche inserite in programmi e reti sovralocali ed internazionali.

La territorialità dei nuovi abitanti risulta inoltre multi localizzata e multi scalare, grazie alla loro frequente appartenenza a reti territoriali lunghe, che uniscono in una relazione biunivoca la Val Maira con i loro luoghi d'origine (in Italia e all'estero) e con altri contesti territoriali interessati da questo nuovo popolamento montano.

\section{La riterritorializzazione delle borgate}

21 Spesso i nuovi abitanti delle aree montane si insediano in località abbandonate $o$ caratterizzate da un'estrema rarefazione sociale e territoriale, creando nuovo presidio territoriale e nuovo territorio laddove quello precedente stava andando perso. In geografia questo può essere letto attraverso il ciclo territorializzazionedeterritorializzazione-riterritorializzazione, proposto da Raffestin (1984). Alla perdita di funzioni da parte di un luogo corrisponde il venire meno delle territorialità che lo connotano. A queste si può sostituire una riterritorializzazione, costituita dall'affermarsi di nuove funzioni del luogo, da una sua nuova attribuzione di valore, di fatto quindi dalla sua trasformazione in un nuovo territorio. In Val Maira questo processo è evidente per esempio nelle vicende della borgata di San Martino inferiore (Stroppo), la quale fino agli anni ' 50 circa era una popolosa borgata agricola come ne esistevano centinaia nella valle, completamente abbandonata e semidiroccata in seguito al completo spopolamento dei decenni successivi, nei primi anni ' 90 , fu scoperta da una coppia austriaco-tedesca che decise di acquistarvi una casa, ristrutturarla ed avviarvi un'attività ricettiva. Da allora San Martino, che oggi ha attirato anche altri nuovi abitanti, è il luogo della Val Maira più conosciuto e frequentato dai turisti di lingua tedesca ed ha sostituito la propria precedente territorialità di borgata alpina rurale, venuta meno in seguito allo spopolamento, con una nuova territorialità legata al suo essere il nodo di una rete internazionale turistica e culturale (figura 2). 
Figura 2. Il processo di riterritorializzazione della borgata di San Martino inferiore (Stroppo)

Territorialità: borgata rurale (fino al 1960 c.ca)

\section{I}

Deterritorializzazione: Declino dell'agricoltura di montagna e progressivo abbandono.

\section{I}

Riterritorializzazione: insediamento dei nuovi residenti ed apertura del centro culturale tedesco



Nuova territorialità: oggi a San Martino vivono diversi nuclei familiari e la borgata attira turisti da tutta Europa

\section{II rallentamento della spirale della marginalità}

22 Tra i fattori principali della spirale negativa che porta un territorio rurale spopolato verso la marginalità economica e sociale vi è il mancato raggiungimento della soglia critica di popolazione tale da giustificare economicamente la presenza di servizi ed attività economiche (Buran et al., 1998). Uno degli impatti maggiori dell'afflusso di nuovi abitanti in età lavorativa in Val Maira è stato senza dubbio l'aumento dell'utenza dei servizi locali e delle attività commerciali, che in alcuni casi, secondo le informazioni raccolte, ha contribuito a garantirne il mantenimento, in particolare per quanto riguarda le scuole e gli esercizi commerciali di prossimità. I nuovi abitanti intervistati hanno dimostrato una consapevolezza del proprio ruolo di protagonisti attivi della vita delle comunità nelle quali si sono insediati, manifestata ad esempio attraverso la scelta esplicita di effettuare dei propri acquisti quotidiani nei pochi negozi della valle, considerati un importante presidio territoriale.

I dati sembrano sostenere questa tesi, se si considera che Stroppo alla fine degli anni ' 90 (Buran et al. 1998) si trovava al quindicesimo posto della classifica dei comuni più marginali del Piemonte dal punto di vista socio-economico, mentre nell'aggiornamento più recente di quell'analisi (Crescimanno et al., 2010), non risulta più nelle ultime venti posizioni. In base alle informazioni raccolte nel corso delle interviste, l'arrivo in Val Maira di nuova popolazione ha contribuito inoltre in modo determinante (anche se naturalmente non esclusivo) a creare sul territorio un tessuto sociale ed economico tale da essere considerato condizione sufficiente per restare a vivere in un comune della valle, da una percentuale di giovani (20-35 anni) originari del luogo molto maggiore rispetto a quanto accadesse fino a dieci o venti anni fa. Questo dato è confermato dai 
dati demografici rilevati dall'ultimo censimento della popolazione (2012), che attribuiscono a tutti paesi dell'alta val Maira un saldo migratorio positivo.

\section{L'importanza dei fattori localizzativi}

Dalle interviste con i nuovi abitanti della Val Maira emerge come alcune caratteristiche del territorio fungano da fattori localizzativi di attrazione. Tra queste una delle principali è costituita dall'amenity della località scelta come nuova residenza: le borgate maggiormente interessate dai fenomeni di riterritorializzazione legati all'insediamento di nuovi abitanti sono infatti quelle collocate sul versante soleggiato della valle, inserite in un contesto ambientale di qualità, non eccessivamente vicine alla strada statale che percorre la valle (anche se l'accessibilità automobilistica è una variabile importante presa in considerazione nella scelta).

Un seconda variabile nella scelta della nuova residenza espressa dagli intervistati è la disponibilità di abitazioni, maggiore nelle borgate più lontane dalla strada principale $\mathrm{e}$ dalla minore vocazione turistica.

Infine, è stata descritta come fondamentale nella localizzazione del nuovo luogo di vita la disponibilità di servizi di base ad una distanza ragionevole, in particolare le scuole primarie e secondarie, un negozio con i generi alimentari e di prima necessità e l'accessibilità alla rete internet.

\section{Conclusioni}

27 La ricerca sugli effetti territoriali della presenza di nuovi abitanti in un territorio montano marginale come la Val Maira necessiterebbe di ulteriori approfondimenti, in primo luogo un confronto strutturato con altre aree $^{9}$ ed il coinvolgimento degli abitanti autoctoni, per analizzare più in dettaglio le differenze e le somiglianze tra le relazioni con il territorio di questi ultimi e quelle dei nuovi insediati.

La consistenza del campione coinvolto nelle ricerche restituite in questo contributo, in relazione alla dimensione demografica della valle, permette tuttavia di offrire alcuni spunti di riflessione conclusivi, considerando, come esplicitato all'inizio dell'articolo, la Val Maira come una sorta di laboratorio territoriale nel quale studiare il fenomeno, dove l'arrivo di nuovi abitanti ha effettivamente determinato, come sottolineato più volte nel corso di questo contributo, un importante cambiamento di rotta, per quanto riguarda sia le dinamiche interne alla valle, sia le sue relazioni con altri territori, montani e non.

\section{Il nuovo popolamento avviene spontaneamente, anche in assenza di politiche adeguate}

29 Il primo aspetto da considerare riguarda le politiche di accoglienza ed incentivo all'insediamento di nuovi abitanti nei comuni montani. In Italia politiche strutturali finalizzate ad attirare nuova popolazione nelle aree montane deboli risultano assenti, salvo alcune sporadiche e poco incisive eccezioni. Ciononostante da almeno vent'anni, in diverse valli dell'intero arco alpino italiano migliaia di persone hanno scelto di trasferirsi a vivere e, dove possibile, lavorare in montagna, ritenendo che questa 
offrisse opportunità migliori rispetto alla città ed alla pianura per i loro progetti lavorativi e personali. Questo anche grazie ad una sorta di "effetto contagio": la riterritorializzazione di alcuni luoghi in una direzione, opposta a quella di una marginalità che sembrava irreversibile, è servita da esempio per altri territori e altre persone, che forti delle buone pratiche esistenti hanno avviato progetti analoghi ed ha contribuito a costituire un tessuto sociale e culturale nuovo, considerato a sua volta dalla maggior parte dei nuovi abitanti di più recente insediamento un importante fattore attrattivo.

\section{Senza i servizi minimi alla popolazione, un nuovo popolamento diffuso e stabile non è possibile.}

Un secondo elemento su cui riflettere riguarda l'offerta di servizi alla popolazione sul territorio. Quasi tutti i soggetti coinvolti nella ricerca si sono rivelati concordi nel ritenere indispensabile per sostenere i propri progetti nel futuro una fornitura minima di servizi, in particolare per quanto riguarda la presenza delle scuole per i propri figli. In un periodo storico di razionalizzazione economica dei servizi che troppo spesso si fonda su criteri del tutto a-territoriali, sembra dunque che senza la garanzia del presidio dei servizi di base, non sia possibile non solo mettere in atto politiche sostenibili di coesione territoriale e contrasto alla marginalità socio-economica delle aree fragili, ma nemmeno sostenere le tendenze spontanee in atto, in grado di portare esternalità positive al territorio montano.

\section{I nuovi abitanti protagonisti di un nuovo rapporto tra montagna e città}

31 Uno dei contributi principali delle relazioni che i nuovi abitanti della valle hanno instaurato con il territorio consiste nel superamento della subalternità - soprattutto culturale - nei confronti della città subita da molte aree montane nell'ultimo secolo. Molti abitanti della Val Maira oggi provengono dalla città e hanno scelto di vivere in montagna perché ritengono che quel contesto territoriale offra risorse migliori alle proprie progettualità personali e lavorative. La montagna per questi ex cittadini non è più solo una periferia marginale o un luogo di svago, ma un territorio di progetto. Questo ribalta la tradizionale (e forse stereotipata) relazione città-montagna, come dimostrano anche i numerosi eventi organizzati dai nuovi abitanti, spesso riuniti in associazione, per riflettere sul futuro della montagna, nei quali la città ed i suoi esperti sono interlocutori invitati e graditi, ma non dominanti.

BIBLIOGRAPHIE

BARTALETTI F., 2004.- Geografia e cultura delle Alpi, Franco Angeli, Milano. 
BÄTZING W., 2005.- Le Alpi. Una regione unica al centro d'Europa, Bollati Boringhieri, Torino. BURAN P. (a cura di), 1998.- Le misure della marginalità. I fattori del disagio territoriale delle aree montane piemontesi, Working Paper $n^{\circ} 121$, Ires Piemonte, Torino.

CAMANNI E., 2002.- La nuova vita delle Alpi, Bollati Boringhieri, Torino.

CHIPENIUK, R., 2004.- « Planning for amenity migration in Canada : current capacities of interior British Columbian mountain communities ", in Mountain Research and Development,vol. 24, ${ }^{\circ} 4$, pp. 327-335.

COGNARD, F., 2006.- « Le rôle des recompositions sociodémographiques dans les nouvelles dynamiques rurales : l'exemple du Diois ", Mediterranée, n³, pp. 5.

CORRADO F., 2011.- « Il fenomeno dei nuovi abitanti nelle Alpi e in Europa », in Dematteis G., Montanari per scelta, pp. 11-21.

CORRADO F. , 2010.- « Les territoires fragiles dans la région alpine : une proposition de lecture entre innovation et marginalité », Revue de géographie alpine, 98-3.

CRESCIMANNO A., FERLAINO F. E ROTA F. , 2010.- La montagna del Piemonte, Ires Piemonte, Torino. DANSERO E., MARONI O., RICCIARDI C., 2003.- « Cercando SLOT per le Valli Chisone e Germanasca », in Rossignolo C. E simonetta c. (a cura di), Una geografia dei luoghi per lo sviluppo locale, Baskerville, Bologna pp. 111-145.

DemAtTeis G. (a cura di), 2011.- Montanari per scelta, Franco Angeli, Milano.

FouRNY M.-Ch., 1994.- « Nouveaux habitants dans un pays de moyenne montagne », Études Rurales, $\mathrm{n}^{\circ} 135-136, \mathrm{pp} .83-95$.

GOLINI A., MUSSINO A., SAVIOLI M., 2000.- Il malessere demografico in Italia, Il Mulino, Bologna GOVERNA F., 2005.- « Sul ruolo attivo della territorialità ", in Dematteis G. e Governa F., Territorialità, sviluppo locale, sostenibilità : il modello SLoT, Franco Angeli, Milano, pp. 39-67. MAGNAGHI, A., 2000.- Il progetto locale, Bollati Boringhieri, Torino. PAINTER J. AND JEFFREY A., 2009.- Political geography, SAGE, Londra. PERLIK M., MESSERLI P. BÄTZING W., 2001.- « Tows in the Alps », Mountain Research and Development, 21, pp. 243-252.

PLA-LANG L., 2008.- Occitano in Piemonte : riscoperta di un'identità culturale e linguistica ?, Lang, Amburgo.

RAFFESTIN C., 1984.- « Territorializzazione, deterritorializzazione, riterritorializzazione e informazione », in turco A. (a cura di), Regione e regionalizzazione, Franco Angeli, Milano, pp. 69-82. Regione Piemonte, 2012.- Piemonte in cifre 2012, Regione Piemonte, Torino.

REVELLI, N., 1977.- Il mondo dei vinti, Einaudi, Torino.

SALSA, A., 2007.- Il tramonto delle identità tradizionali, Priuli e Verlucca, Torino.

TURRI E., 2004.- La megalopoli padana, Marsilio, Venezia.

VIAZZO P., 1989.- Upland Communities. Environment, Population and Social Structure in the Alps since the Sixteenth Century, Cambridge University Press, Cambridge.

ZANZI L., 2004.- Le Alpi nella storia d'Europa, CDA\&Vivalda, Torino. 


\section{NOTES}

1. L'inizio delle mie ricerche sul tema del nuovo popolamento della Val Maira è coinciso con la realizzazione di una tesi di laurea in Scienze Geografiche (prof. Egidio Dansero), alla quale hanno fatto seguito altre ricerche sugli stessi temi, organizzate dall'Associazione Dislivelli di Torino (ricerca Novalp, in corso) e dal Coordinamento Donne di Montagna, con sede in Val Maira.

2. In Francia lo spopolamento interessa le Alpi a partire dal 1860 circa, mentre sul versante italiano questo fenomeno si presenta negli anni '30 del XX secolo (Bätzing, 2005).

3. Non si intende prendere in considerazione, in questo contributo, il ripopolamento dei comuni alpini che possono essere considerati periurbani, riconducibile a quella che Bätzing (2005) definisce la "metropolizzazione" della montagna, presente sia sul versante francese (Barthes, 2006), che su quello italiano (Turri 2004, per una riflessione su tutto l'arco alpino si veda invece Perlik et al., 2001).

4. Si veda a questo proposito anche la relazione finale del progetto Interreg IVC PADIMA Policies Against Depopulation in Mountai Areas, intitolata "Strategies to increase the attractiveness of mountain areas: how to approach depopulation in an integrated manner?"

5. La legislazione italiana è confusa in merito alla definizione ufficiale del territorio montano. Qui si fa riferimento alle classificazioni utilizzate da Ires Piemonte (Crescimanno et al., 2010), incrociando le definizioni legislative con le ripartizioni statistiche.

6. L'idea delle Alpi come "il più qualificato laboratorio in cui sperimentare uno sviluppo alternativo" proviene, tra gli altri, da Camanni (2002, p. 99)

7. Non è questa la sede per approfondire il concetto di territorialità, molto dibattuto nella disciplina geografica. La si intenda qui come "modi materiali e non di rapportarsi al territorio" (Dansero et al., 2003)

8. Da questo punto di vista, i casi più interessanti sono probabilmente quelli del Coordinamento Donne di Montagna e dell'Associazione Alte Terre.

9. Le attività di ricerca dell'Associazione Dislivelli vanno in questa direzione (ricerca NOVALP).

\section{RÉSUMÉS}

Le Alpi occidentali italiane, rese marginali socialmente, culturalmente ed economicamente da un lungo periodo di emigrazione, a partire dagli anni ' 80 vivono quello che si può definire un nuovo popolamento. Sono sempre più numerosi, infatti gli esempi di nuclei familiari e singoli cittadini provenienti dalla città e dalla pianura che si trasferiscono in montagna, dove mettono in atto progetti personali e lavorativi spesso caratterizzati da interessanti elementi di innovazione. Questo contributo si propone di approfondire il ruolo dei nuovi abitanti nell'indirizzare le dinamiche sociali ed economiche della Val Maira, considerata per la consistenza e la rilevanza del fenomeno un "laboratorio territoriale" di particolare interesse, a partire dai risultati di una serie di ricerche condotte tra il 2008 ed il 2013. Attraverso 36 interviste con i nuovi abitanti della valle si è potuto verificare come le loro relazioni con il territorio montano siano particolarmente significative, soprattutto grazie al fatto che esso si trova al centro dei loro progetti di vita. Questo ha determinato una riterritorializzazione positiva di molte aree della valle, impoverite dallo spopolamento e dalla perdita di funzioni ed un rallentamento del processo di marginalizzazione socio-economica. Allo stesso tempo emerge come questo fenomeno esclusivamente spontaneo 
necessiti di politiche efficaci di sostegno, affinché sia sostenibile nel tempo ed in grado di modificare realmente le dinamiche territoriali.

INDEX

Keywords : Alpi italiane, Piemonte, nuovi abitanti, marginalità, sviluppo locale

\section{AUTEUR}

\section{GIACOMO PETTENATI}

Ambiente e Territorio - Pianificazione Territoriale e Sviluppo Locale, Dipartimento Interateneo di Scienze, Progetto e Politiche del Territorio, Politecnico di Torino giacomo.pettenati@polito.it 